\title{
New Stereology for the Recovery of Grain-Boundary Plane Distributions in the Crystal Frame
}

\author{
Brent L. Adams \\ b_I_adams@byu.edu \\ Ryan J. Larsen
}

Follow this and additional works at: https://scholarsarchive.byu.edu/facpub

Part of the Mechanical Engineering Commons

Original Publication Citation

Metallurgical and Materials Transactions Jul 24 35A, 7 Research Library pg. 1991

\section{BYU ScholarsArchive Citation}

Adams, Brent L. and Larsen, Ryan J., "New Stereology for the Recovery of Grain-Boundary Plane Distributions in the Crystal Frame" (2004). Faculty Publications. 426.

https://scholarsarchive.byu.edu/facpub/426 


\title{
New Stereology for the Recovery of Grain-Boundary Plane Distributions in the Crystal Frame
}

\begin{abstract}
RYAN J. LARSEN and BRENT L. ADAMS
A new experimental method is given for recovering the probability-distribution function $S_{1}\left(\hat{n}_{1} \mid \Delta g\right)$. The function $S_{v}\left(\hat{n}_{\lambda} \mid \Delta g\right)$ is the grain-boundary area per unit volume as a function of grain-boundary plane orientation $\left(\hat{h}_{A}\right)$, given a lattice misorientation $(\Delta g)$ between the adjoining grains. The grain-boundary normal $\left(\hat{n}_{A}\right)$ is expressed in the crystal frame in which the misorientation $\Delta g$ originates. The proposed method recovers the three-dimensional $S_{v}\left(\hat{n}_{A} \mid \Delta g\right)$ function using data taken from two-dimensional section planes. 'The method requires the measurement of many grain-boundary trace (in-plane) angles and lengths associated with grain boundaries of lattice misorientation. All such boundary traces may be observed from a single section plane if the crystallographic texture is sufficiently random. In heavily textured microstructures, the method requires the researcher to observe traces from multiple oblique section planes cut through the material. A method of quantitatively estimating whether the texture is sulficiently random is given. Simulations on both textured and nontextured microstructures demonstrate the validity of the method. Experimentally, the new method is used to analye boundaries of misorientation (2'3) observed in 304 stainless steel. Calculated grain-boundary plane-probability lunctions are shown to be consistent with what is already known.
\end{abstract}

\section{INTRODUCTION}

CONSIDERABLE research effort in recent years has focused on designing materials that contain large numbers of boundaries with special properties. These special properties include resistance to corrosion, cavitation, or precipitation. Given the difficulty of recovering grain-boundary plane orientations, most current studics characterize grain boundaries only by the relative misorientation between the two grains adjoining the boundary. Although advances have been made by only considering misorientation, simulations and experiments have shown that the orientation of the grainboundary plane is important in determining grain-boundary properties. 11.21

The orientations of grain-boundary planes are often neglected, because current methods for recovering plane orientations (such as serial sectioning and transmission electron microscopy studies) are too time consuming to allow the researcher to gather data sets that are large enough to be statistically reliable. In order to obtain approximations of the boundary orientation distribution, one may resort to the stercological methods of Hilliard ${ }^{\mid 31}$ or Adams. ${ }^{141}$ Both methods synthesize grain-boundary data taken from oblique section planes. However, these methods are designed to recover boundary distributions in a laboratory coordinate frame, which is only useful in grain-boundary design problems that involve a macroscopic field or directionality.

RYAN J. LARSEN, formerly Master's Degree Candidate, Department of Mechanical Fngineering, Brigham Young University, is Doctoral Candidate, Division of Fnginecring and Applicd Sciences. Harvard University, Cambridge, MA (21.39. BREN'T L. ADAMS, Dusenberry Professor, is with the Department of Mechanical Engineering, Brigham Young University, Provo, UTT 84602-4201. Contact e-mail: b___adams@byu.edu

This article is based on a presentation made at the symposium "Characterization and Representation of Material Microstructures in 3-D" held October 8-10,2002, in Columbus, OH, under the auspiecs of ASM International's Phase Transtormations committee.
In most research applications, the grain boundary is expressed in a reference trame embedded in an adjacent erystal. Knowledge of the grain-boundary orientation in this frame is useful because it contains information about the arrangement of atoms near the boundary, thus helping the researeher characterize boundary energy and other properties.

For most rescarchers, the probability distribution function of interest, first described by Adams, ${ }^{1+1}$ is $S_{1}\left(\hat{n}_{A} \mid \Delta g\right)$, or the grain-boundary area per unit volume als a function ol boundary normal $\left(\hat{n}_{1}\right)$ for a given misorientation $(\Delta g)$. For a general misorientation of $\Delta g$, the domain of $\hat{n}_{A}$ is a hemisphere. ${ }^{151}$ Therefore, for consistency, $\hat{n}_{A}$ must be expressed in a standardized crystal reference frame or fundamental zone, which we here call the misorientation frame. Since the misorientation frame of a given bicrystal in a sample has a known orientation with respect to the laboratory frame, we call it a local misorientation frame (LMF). However, since $S^{\prime}\left(\hat{n}_{1} \mid \Delta g\right)$ is an average of grain-boundary plane orientations taken from many bicrystals, it contains no information relating grain-boundary plane distributions to the laboratory frame. We, therefore, saly that the function $S_{1}\left(\hat{n}_{\lambda} \mid \Delta g\right)$ is expressed in the generalized misorientation frame (GMl). The conceptual distinction between LMFs and GMFs is fundamental to crystallite stereology.

The goal of erystallite stereology is to estimate $S_{1}\left(\hat{n}_{A} \mid \Delta g\right)$ by observing large numbers of boundary traces on twodimensional data sets. If the texture of the observed material is random, the macroscopic distribution of I MF's will be random. If a single section plane is large enough to expose multiple bicrystals of misorientation $\Delta g$, then each observed bicrystal provides a unique "view" of a grain boundary associated with $\Delta g$. Each view consists of a grain-boundary trace (Figure 1). Since the boundary normal must be perpendicular to the observed trace, the measurement provides partial information about the orientation of the grain-boundary plane. By piecing together this partial information from numerous observed traces, the researcher may arrive at an estimate of $S_{v}\left(\hat{n}_{\Lambda} \mid \Delta g\right)^{|6.9|}$ 


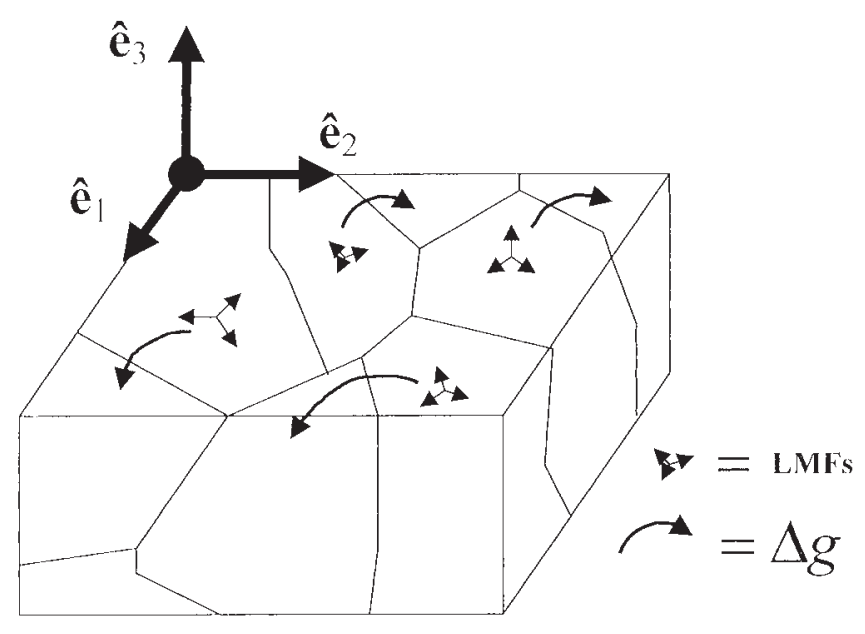

Jïg. 1-Various I.MFis, which have different orientations with respect to the laboratory frame.

The current article develops a new stereology for the recovery of $S_{i}\left(\hat{n}_{a} \mid \Delta g\right)$. Simulations demonstrate that the proposed method recovers accurate function values. Furthermore, recoveries of $S_{n}\left(\hat{n}_{A} \mid \sum 3\right)$ from actual data sets show trends consistent with the work of others.

This new stereology is a powerful tool that promises to provide many insights into the preferred orientations of the grain-boundary plane, to help researchers measure how the grain-boundary plane character changes during processing and to better determine the extent that the grain-boundary plane influences the macroscopic properties of the material. It is our hope that this new approach will foster a more complete approach to grain-boundary engineering and design.

\section{THEORETICAL}

\section{A. The Domain of $S_{1}\left(\hat{n}_{\lambda} \mid \Delta g\right)$}

Before presenting the new method of solving for $S_{n}\left(\hat{n}_{\Lambda} \mid \Delta g\right)$, we briefly review the procedure for finding the fundamental zone (or misorientation frame) and discuss some complications imherent in plotting $S_{v}\left(\hat{h}_{\lambda} \mid \Delta g\right)$ duc 10 special symmetries.

\section{Misoricutation- $\Delta g$}

In order to deline the misorientation frame, we must fïrst deline the misorientation. In agreement with Bunge, ${ }^{[10 \mid}$ the misorientation from grain A to grain B is given by

$$
\Delta g=c_{i} g_{B} g_{A}^{T} c_{j}^{T}
$$

where $g_{A}$ and $g_{B}$ are three-by-three matrices of direction cosines linking the lattice orientations of grain A and grain B to the laboratory frame, and the $c_{i}$ values are the complete set of proper symmetry operators corresponding to the symmetry group of the crystal lattice. All misorientations given by Eq. [1] have the same physical meaning in the crystalline world.

\section{The misorientation frame}

The method for defining the misorientation frame is simply a matter of isolating a single fundamental zone from all possible representations of the misoricntation. Fundamental zones of misoricntations in Rodrigues space have been described by Morawiec and Ficld. ${ }^{111}$
The misorientation frame is simply the placement of coordinate frames on grain A and grain B such that the axis of minimum rotation, or the disorientation axis $(\hat{n})$, falls into a zone in grain A given by $\hat{n}_{x}>\hat{n}_{x}>\hat{n}_{x}>0$. This zone forms the familiar "standard triangle" of crystal directions in a stereographic projection.

Using this procedure, the designation of grain $\mathrm{A}$ is generally unique. This unique designation of grain $\mathrm{A}$ is useful in avoiding error in the representation of the function $S_{v}\left(\hat{n}_{A} \mid \Delta g\right)$. If $S_{v}\left(\hat{n}_{A} \mid \Delta g\right)$ is known, a companion function, $S_{v}\left(\hat{n}_{l s} \mid \Delta g^{T}\right)$, can be plotted from the relationship $\hat{n}_{l}=\Delta g \hat{n}_{\lambda}$. Here, $\hat{n}_{k}$ gives the coordinates of the boundary normal in the crystal coordinate frame associated with grain $B$.

The given method of distinguishing $\Delta g$ from $\Delta g^{T}$ (and, thus, $\hat{n}_{A}$ from $\hat{n}_{l B}$ ) does not hold when the axis of disorientation for $\Delta g$ falls on the perimeter of the standard triangle. Although it is highly unlikcly that the calculated axis of disorientation will fall exactly on this perimeter, given the experimental crror of the measurements and misorientation variations in the crystals, there will be a zone about the perimeter in which any practical distinction between $\Delta g$ and $\Delta g^{T}$ will not exist. In these cases, peaks corresponding to both $\hat{n}_{A}$ and $\hat{n}_{B}$ will likely appear in a plot of $S_{1^{\prime}}\left(\hat{n}_{A} \mid \Delta g\right)$. This effect should be considered when interpreting $S_{v}\left(\hat{n}_{A} \mid \Delta g\right)$ for these special misorientations.

When the axis of disorientation is an $n$-fold axis of crystal symmetry, the domain of $\hat{n}_{A}$ in the function $S_{V}\left(\hat{n}_{A} \mid \Delta g\right)$ shrinks (in general) by a factor of $n \cdot{ }^{[2]}$ Equivalently, the distribution in the original domain has $n$-fold symmetry. The exception to this occurs in centrosymmetric crystals when the angle of rotation about the $n$-fold symmetry axis is $\pi / n$, in which case the domain of $\hat{n}_{A}$ shrinks by a factor of $2 n$.

For example, consider a $\Sigma 3$ misorientation (or 60 deg about a $[111]$ axis). Since the $[111]$ axis is an axis of threcfold symmetry in centrosymmetric cubic crystals, the boundary plane distribution must have at least threefold symmetry. Moreover, since the angle of rotation is $60 \mathrm{deg}$, there will be six fold symmetry about $[111]$ in the distribution of $\hat{n}_{A}$ in the hemisphere.

\section{B. Assumptions Required for Derivation of the Fundamental Equation}

The derivation of the fundamental equation of the new $S_{v}\left(\hat{n}_{A} \mid \Delta g\right)$ stereology requires two assumptions: (1) that the collected traces sample the entire population of grain-boundary planes associated with $\Delta g$ in a statistically representative fashion and (2) that the traces sampled from each boundary type are randomly chosen with no orientation bias.

It is clear that when a sample has a completely random texture, both conditions may be satisfied by traces collected from a single section plane. In this case, the LMFs are randomly oriented with respect to the laboratory frame. However, when the material is textured, there will be sampling bias when a single-section plane is used for $S_{v}\left(\hat{n}_{\lambda} \mid \Delta g\right)$ recovery. This bias may be overcome by obtaining traces from several oblique section planes, as demonstrated by simulations shown on Table II.

In order to estimate whether or not a given material is sufficiently random for the stereology to be performed, we introduce the probability-distribution function $C_{n}\left(\hat{n}_{\Lambda} \mid \Delta g\right)$. The function $C_{n}\left(\hat{n}_{A} \mid \Delta g\right)$ is the distribution of scction-plane nor- 
mals in the GMF. The function $C_{n}\left(\hat{n}_{A} \mid \Delta g\right)$ shares the same domain as $S_{n}\left(\hat{n}_{A} \mid \Delta g\right)$, since both are distributions of plane normals in the GMF. Unlike $S_{v}\left(\hat{n}_{n} \mid \Delta g\right), C_{n}\left(\hat{n}_{A} \mid \Delta g\right)$ is easy to obtain experimentally because it involves representing each section-plane normal in the local crystal frame, or LMF, of each bicrystal cut by that section plane. The value of $C_{n}\left(\hat{n}_{1} \mid \Delta g\right)$ is then the distribution of all such plane normals as if they were associated with a single crystal frame, the GMF. Notice how even a single section plane can give rise to a uniform distribution in $C_{n}\left(\hat{n}_{A} \mid \Delta g\right)$, as long as the LMF's orientations of bicrystals cut by that section plane are randomly oriented. If $C_{n}\left(\hat{n}_{\lambda} \mid \Delta g\right)$ collected from a single section plane is not uniform due to sample texturing, the researcher should cut additional oblique section planes. Data from the additional section planes will sample the boundaries more randomly, and this will be reflected in a more uniform $C_{n}\left(\hat{n}_{A} \mid \Delta g\right)$ value. Thus, the uniformity of $C_{n}\left(\hat{n}_{A} \mid \Delta g\right)$ is a simple way to quantitatively estimate the sampling uniformity with respect to the GMF.

We wish to identify a single parameter that will quantify the uniformity of $C_{n}\left(\hat{h}_{A} \mid \Delta g\right)$. We do this by representing $C_{n}\left(\hat{n}_{A} \mid \Delta g\right)$ in terms of surface spherical harmonics according to

$$
C_{n}\left(\hat{n}_{\lambda} \mid \Delta g\right)=\sum_{r=0}^{R} \sum_{i=-r}^{r} C_{r}^{t} K_{r}^{t}\left(\hat{n}_{A}\right)
$$

where the functions $K_{r}^{\prime}\left(\hat{n}_{\lambda}\right)$ are surface spherical harmonics, and the $C_{r}^{\prime}$ functions are weighting coefficients, obtained from the data in the usual fashion. The uniformity of $C_{n}\left(\hat{n}_{1} \mid \Delta g\right)$ is estimated by summing the moduli of the low-order coefficients in the series expansion. The uniformity estimator $\left\langle C_{n}\right\rangle$ is given by

$$
<C_{n}>=\sum_{m=-2}^{2} C_{2}^{m m} \cdot C_{2}^{m}
$$

Because the function $C_{n}\left(\hat{n}_{\Lambda} \mid \Delta g\right)$ is even, the five $C_{2}^{\prime \prime}$ coefficients in Eq. [3] are the lowest-order coefficients associated with nonconstant basis functions. Low $\left\langle C_{n}\right\rangle$ values correspond to uniform distributions of $C_{n}\left(\hat{n}_{A} \mid \Delta g\right)$. For $C_{n}\left(\hat{n}_{A} \mid \Delta g\right)$ distributions generated with pseudorandom numbers over the hemisphere, $\left\langle C_{n}\right\rangle$ values typically fall between 0.001 and 0.003 . For reduced domains of $C_{n}\left(\hat{n}_{A} \mid \Delta g\right)$ corresponding to special misorientations, values of $\left\langle C_{n}\right\rangle$ are typically smaller.

The usefulness of $\left\langle C_{n}\right\rangle$ in estimating whether the collected traces are sufficiently random is established by the simulations on textured materials. We note, however, that the only way for a researcher to be absolutely certain that the aforementioned two assumptions are satisfied is to obtain a full three-dimensional characterization of the microstructure.

\section{Derivation of Fundamental Equation}

Consider a volume of material that has been sampled by one or more section planes, such that both assumptions given in Section II-B are satisfied. In order to draw a quantitative relation between the distribution of observed traces in the GMF and $S_{r} \cdot\left(\hat{n}_{A} \mid \Delta g\right)$, we first recognize that the normal $\left(\hat{n}_{A}\right)$ of the grain boundary associated with a trace vector $(\hat{t})$ must lie on the great circle perpendicular to $\hat{t}$. Since two parameters are needed to quantify the orientation of $\hat{n}_{A}$, knowledge of the orientation of any trace from the bound- ary reveals one of the parameters, leaving only one unknown parameter. Knowing this, it is natural to express a known trace vector in a coordinate frame that decouples the known and unknown parameters. This is done by representing the trace vector in a new coordinate system, $\hat{e}_{i}^{S}$, in which the trace vector lies in the $\hat{e}_{i}^{S}-\hat{e}_{2}^{S}$ plane. If the race makes an angle of $\omega^{\prime}$ with respect to the $\hat{e}_{i}^{\prime}$ axis, the normal of the grainboundary plane associated with this trace must have coordinates $(v, \omega)$ where $\omega=\omega^{\prime}+\pi / 2$ (Figure 2). Note how in this coordinate system, the arimuthal angle $(\omega)$ of the boundary normal is known and the polar angle of the boundary $(v)$ is unknown and independent of $\omega$.

We generally expect that for any given $\hat{e}_{i}^{S}$ coordinate system, there will be a subpopulation of observed trace vectors that are nearly perpendicular to the $\hat{e}_{3}^{S}$ direction. We define "nearly perpendicular" to mean those traces which have angular deviations less than $\varepsilon$ from perpendicularity to the $\hat{e}_{3}^{S}$ axis, where in the current study we have set $\varepsilon=3 \mathrm{deg}$. If we make the approximation that all such traces are exactly perpendicular to the $\hat{e}_{3}^{s}$ direction, we may describe their distribution in the $\hat{e}_{i}^{S}-\hat{e}_{2}^{S}$ plane with the probability-density function $I_{A}\left(\omega \mid \Delta g, \hat{e}_{3}^{s}\right)$. Note that $\omega$, the independent variable of $l_{\lambda}$, is the angle between the trace normal and the $e_{i}^{s}$ axis, as shown in Figure 2. The function $l_{A}\left(\omega \mid \Delta g, \hat{\rho}_{3}^{S}\right)$ has units of trace length per unit area. The area used to normalize $f_{A}\left(\omega \mid \Delta g, \hat{e}_{3}^{s}\right)$ is the total scanned area from which all observed traces were collected.

We are now in a position to write the fundamental equation relating $l_{\Delta}\left(\omega \mid \Delta g, \hat{e}_{3}^{S}\right)$ to $S_{1}\left(\hat{n}_{A} \mid \Delta g, \hat{e}_{3}^{S}\right)$ in any $S$-coordinate frame. (Note that in the fundamental equation, $S_{v}\left(\hat{n}_{A} \mid \Delta g\right)$ is expressed in the $S$ frame as $\left.S_{1}\left(\hat{n}_{A} \mid \Delta g, \hat{o}_{3}^{S}\right)\right)$. The fundamental equation is given by

$$
I_{A}\left(\omega \mid \Delta g, \hat{e}_{3}^{S}\right)=\frac{\pi}{4} \int_{0}^{\pi / 2} S_{1}\left(v, \omega \mid \Delta g, \hat{e}_{3}^{S}\right) P(v) \sin (v) d v|4|
$$

where $P(v)$ is the probability that a trace randomly selected from a grain boundary of polar angle $v$ in the $S$ frame will

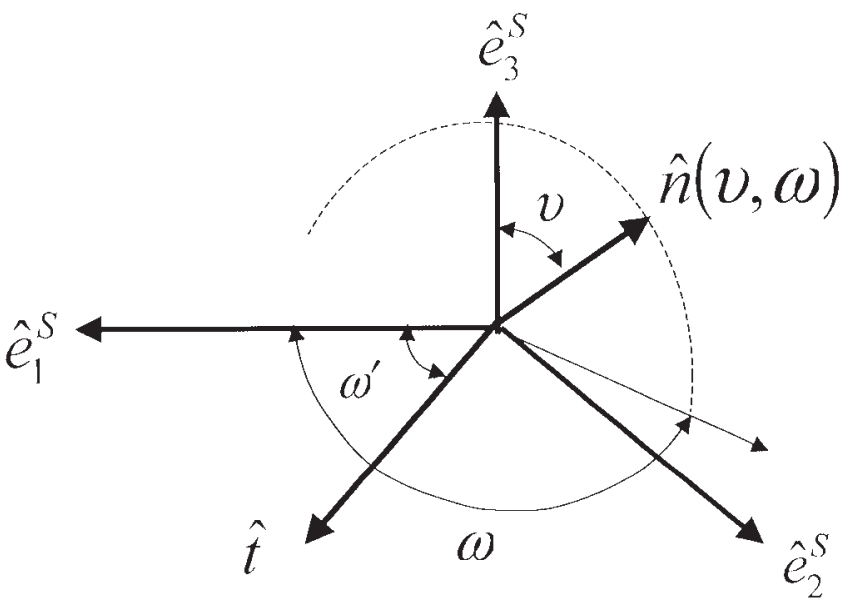

Fig. 2-A ramdom trace $\hat{f}$ laken from a grain boundary of normal $\hat{n}$ is expressed in an $S$ coordinate frame, $\hat{e}_{i}^{S}$, such thall the trace lices in the $\hat{r}_{1}^{S}-\hat{e}_{2}^{S}$ frame and forms an angle $\omega^{\prime}$ with the $\hat{c}^{S}$ axis. The boundary mormal $\hat{n}$ monst have coordinates $(v, \omega)$ in the $S$ frame, where $\omega: \omega^{\prime}+\pi / 2$. Notice how the armuthal coordinate, $\omega$, is constrained once the race is specified, whereas the polar coordinate, $v$, is independent of the tratce angle. 
have an angular deviation less than $\varepsilon$ from perpendicularity to the $e_{3}$ axis (Figure 3). The value of $P(v)$ is given by

$$
\begin{aligned}
& P(v)=1 \quad \text { when } 0 \leq v \leq \varepsilon \\
& P(v)=\frac{2}{\pi} \sin \cdots\left(\frac{\sin (\varepsilon)}{\sin (v)}\right) \text { otherwise }
\end{aligned}
$$
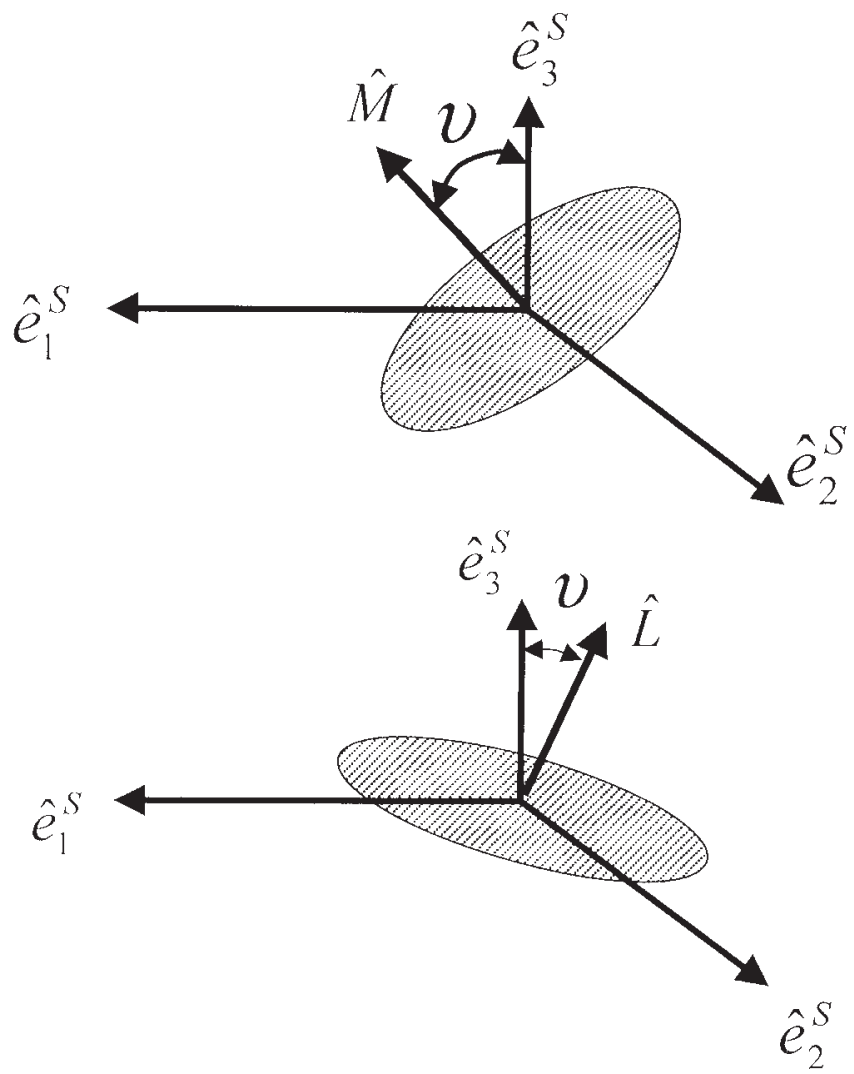

(a)

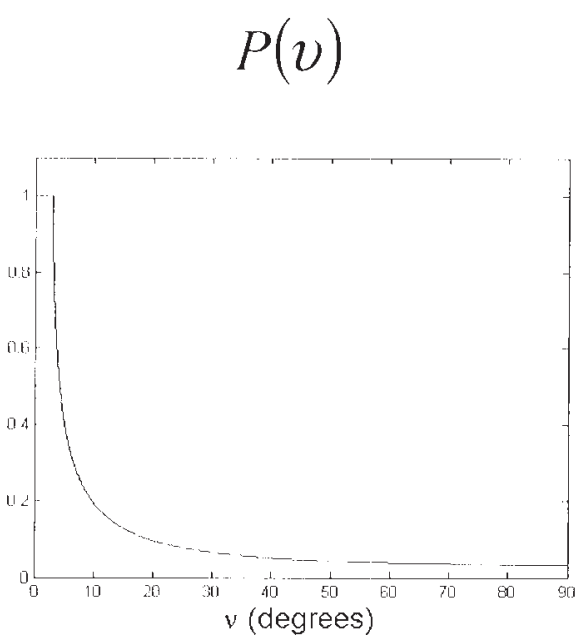

(b)

Fig. 3-(a) A randomly selected trace from plane $\hat{M}$ is less likely to fall within 3 deg of perpendicularity $60 \hat{e}_{3}^{S}$ than a randomly selected trace from plane $\hat{L}$. This is quantified (b) by the probability $P(v)$ for $\varepsilon=3$ deg. $P(v)$ is the probability that a trace rathdomly selected from a grain boundary of polar angle $v$ in the $S$ frame will have an angular deviation less than $\varepsilon$ from perpendicularity to the $e_{3}^{S}$ axis.
In deriving $P(v)$ (refer to the Appendix), it was assumed that all possible traces of a grain boundary have an equal probability of being chosen (Assumption 2). The fundamental relation, Eq. $|4|$, involves a single integration over the single unknown parameter, $v$, weighted by $P(v)$. The lactor $\pi / 4$ is the constant of proportionality between $l_{A}$ and $S_{V}{ }^{|13|}$ These functions are proportional due to representative sampling of all boundary types (Assumption 1).

Equation |4| represents a classic inverse, or ill-posed, problem in which the known function, $l_{A}(\omega \mid \Delta g, \hat{e})_{3}^{S}$, contains only a fraction of the information contained in the unknown function, $S_{1}\left(\hat{n}_{A} \mid \Delta g, \hat{e}_{3}^{S}\right)$. However, we use Eq. |4] to estimate $S_{v}\left(\hat{n}_{A} \mid \Delta g\right)$ by considering the functions $I_{A}\left(\omega \mid \Delta g, \hat{e}_{3}^{S}\right)$ from many $S$ frames. In the current study, we have chosen an array of $S$ frames that have the characteristic that the $\hat{e}_{3}^{S}$ directions sample the domain of $S_{1^{\prime}}\left(\hat{n}_{A} \mid \Delta g\right)$ as uniformly as possible. Fundamental equations from each $S$ frame combine as a system of equations that is solved simultancously to estimate $S_{1}\left(\hat{n}_{A} \mid \Delta g\right)$.

One can create numerous possible methods of solving these fundamental equations. We have chosen to solve the equations using a method proposed by Adams that makes use of surface spherical harmonics. ${ }^{|4|}$ In this method, the distribution functions are represented using surface spherical harmonics and Fourier series according to

$$
\begin{aligned}
S_{1}\left(\hat{n}_{A} \mid \Delta g\right) & =\sum_{r=0}^{R} \sum_{l=1}^{r} S_{r}^{i} K_{r}^{i}\left(\hat{n}_{\Lambda}\right) \\
j_{\Lambda}(\omega) & =\sum_{i=-\infty}^{R} l_{i}^{i} e^{i t(\omega)}
\end{aligned}
$$

where $R$ is the truncation order of the series. Adams obtained matrix equations relating the coefficients $S_{r}$ to the coefficients $l i$ by inserting Eqs. $\mid 6]$ and $[7 \mid$ into a fundamental equation similar to Eq. $|4|$ and performing the proper coordinate transformations from the $S$ frames to the GMF $\left(S_{1}\left(\hat{n}_{A} \mid \Delta g, \hat{e}_{3}^{s}\right)\right.$ to $\left.S_{1}\left(\hat{n}_{A} \mid \Delta g\right)\right)$. As more $S$-coordinate systems are added, the systems of equations become overdetermined, allowing a solution of the coefficients $S_{\text {. }}$ using singular-value decomposition. ${ }^{|4|}$

\section{Alternative Form of the Fundamental Equation}

The concepts presented in the derivation of the fundamental equation may also be applied to recover the function $S_{s}\left(\hat{n}_{A} \mid \Delta g\right)$, or the grain-boundary area fraction. This is simply a matter of replacing $l_{\Lambda}\left(\omega \mid \Delta g, \hat{e}_{3}^{5}\right)$, which is normalized by the total scan area, with $l_{l}\left(\omega \mid \hat{e}_{3}^{s}, \Delta g\right)$, which is normalized by the total trace length. In this case, the fundamental equation is given by

$$
l_{l}\left(\omega \mid \Delta g, \hat{e}_{3}^{S}\right)=\int_{0}^{\pi / 2} S_{s}(v, \omega \mid \Delta g) P(v) \sin (v) d v
$$

\section{E. Simulations}

Simulations were performed to demonstrate that the technique gives viable estimates of $S_{3}\left(\hat{n}_{A} \mid \Delta g\right)$ in both nontextured (Table I) and textured (Table II) microstructures. Simulated microstructures are not three-dimensional arrangements of polyhedra; rather, they are enscmbles of bicrystals with fixed 
Table I. Simulations on Nontextured Microstructures (Random I,MFs)

\begin{tabular}{|c|c|c|c|c|c|c|c|c|}
\hline \multicolumn{2}{|c|}{ Microstructure } & \multicolumn{3}{|c|}{ Recovery } & \multicolumn{4}{|c|}{ Results } \\
\hline $\begin{array}{l}\text { (a) Number } \\
\text { of Habit } \\
\text { Planes }\end{array}$ & $\begin{array}{l}\text { (b) Pet } \\
\text { Random } \\
\text { GBs }\end{array}$ & $\begin{array}{l}\text { (c) Number } \\
\text { of Bicrystals }\end{array}$ & (d) Order R & $\begin{array}{c}\text { (e) Trace } \\
\text { Measured } \\
\text { Error }\end{array}$ & $\begin{array}{c}\text { (l) Pct ol HPs } \\
\text { with <10 Pet } \\
\text { Error }\end{array}$ & $\begin{array}{c}\text { (g) Pct of } \mathrm{HPs} \\
\text { with }<30 \mathrm{Pct} \\
\text { Error }\end{array}$ & $\begin{array}{l}\text { (h) Mean } \\
\text { Eirrol }\end{array}$ & $\begin{array}{l}\text { (i) Number } \\
\text { of lialse } \\
\text { Peaks }\end{array}$ \\
\hline \multicolumn{9}{|c|}{ General Misorientation-No Symmetry } \\
\hline $\begin{array}{l}8 \\
4\end{array}$ & $\begin{array}{l}20 \mathrm{pct} \\
30 \mathrm{pct}\end{array}$ & $\begin{array}{r}300 \\
1000 \\
\end{array}$ & $\begin{array}{l}34 \\
24 \\
\end{array}$ & $\begin{array}{r}0 \\
\pm 5 \mathrm{deg} \\
\end{array}$ & $\begin{array}{l}49 \mathrm{pct} \\
16 \mathrm{pct}\end{array}$ & $\begin{array}{r}93 \mathrm{pct} \\
100 \mathrm{pct}\end{array}$ & $\begin{array}{l}13 \mathrm{pet} \\
15 \mathrm{pet}\end{array}$ & $\begin{array}{l}4 / 400 \\
0 / 200\end{array}$ \\
\hline \multicolumn{9}{|c|}{ ¿3 Misorientation-Sixfold Symmetry } \\
\hline $\begin{array}{l}7 \\
2\end{array}$ & $\begin{array}{l}20 \mathrm{pct} \\
70 \mathrm{pct}\end{array}$ & $\begin{array}{r}500 \\
1000 \\
\end{array}$ & $\begin{array}{l}34 \\
24 \\
\end{array}$ & $\begin{array}{r}0 \\
+5 \mathrm{deg} \\
\end{array}$ & $\begin{array}{l}39 \mathrm{pct} \\
32 \mathrm{pct} \\
\end{array}$ & $\begin{array}{l}85 \mathrm{pet} \\
87 \mathrm{pet}\end{array}$ & $\begin{array}{l}16 \mathrm{pet} \\
16 \mathrm{pet}\end{array}$ & $\begin{array}{r}16 / 350 \\
0 / 100 \\
\end{array}$ \\
\hline \multicolumn{9}{|c|}{ 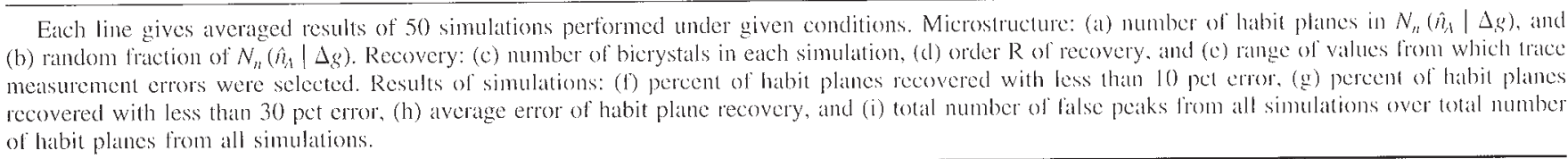 } \\
\hline
\end{tabular}

Table II. Simulations on Textured Material

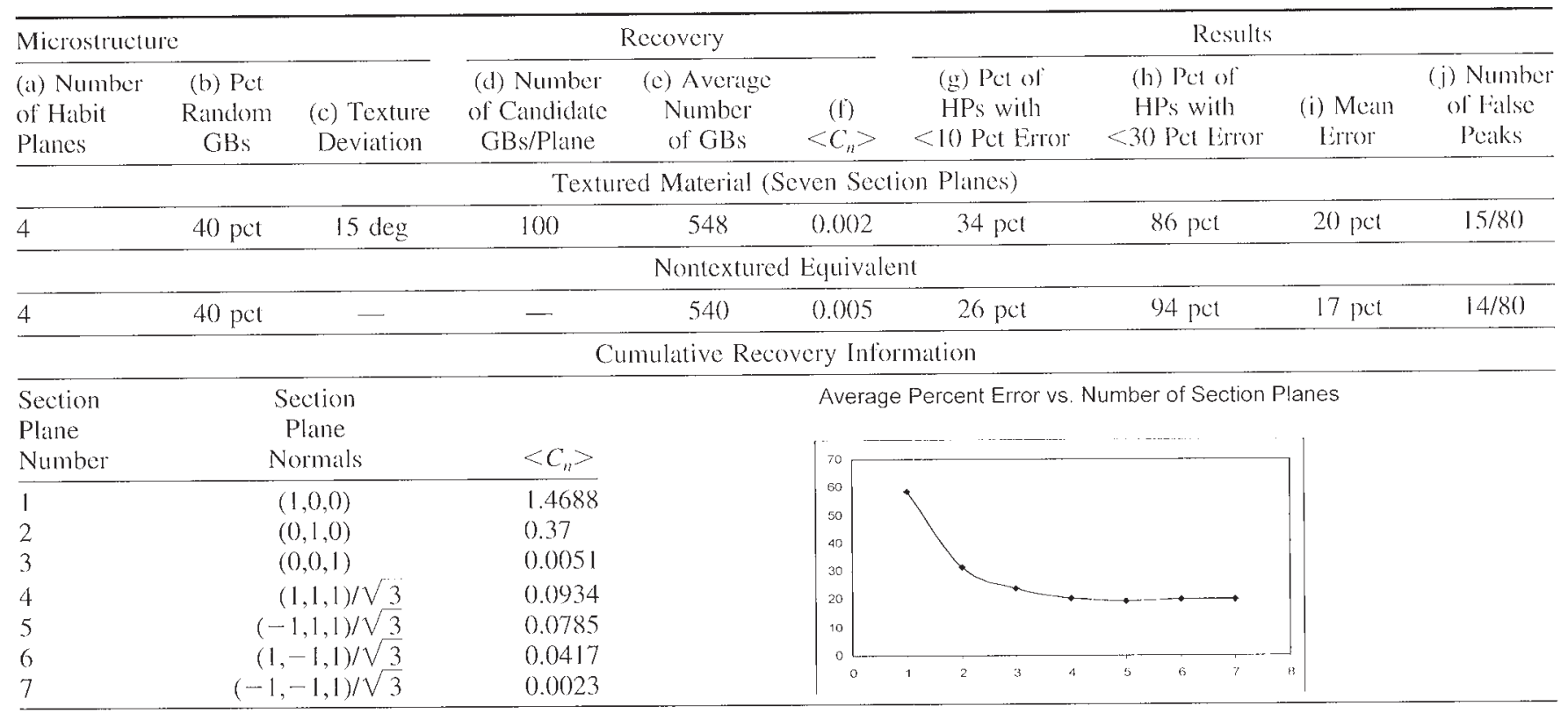

The lirst line gives the averaged results of 20 simulations performed under the given conditions. 'The second line gives the results of 20 simulations performed on nontextured microstructures with otherwise similar conditions. Conditions are as follows. All textured simulations were performed using the seven section planes shown above. Microstructure: (a) number of habit planes in $N_{n}\left(\hat{n}_{\Lambda} \mid \Delta g\right)$, (b) pandom fraction of $N_{n}\left(\hat{n}_{A} \mid \Delta g\right)$, and (c) maximum allowed angular devialion of L.Mli orientations from the central LMI: orientation. Recovery: (d) number of candidate bicrystals for each section plane; (c) number of observed boundaries from all section planes, averaged over all simulations; and $(f)<C_{n}>$ from all section planes, alveraged over all simulations. Order of recovery lor all simulations wis $R$ : 24 with trace neastrement error range of \pm 5 deg. Results: (g) percent of habit planes (HPs) recovered with less than 10 pet error, (h) perecnt of hatbit planes recovered with tess than 30 pet error, (i) average error of habit plane recovery, and (j) total number of false peaks from all simulations over total number of habit planes from all simulations. The bottom section of the table shows the cumulative results of recoveries pertormed as each section plane was added. Columns on the lefi show plane nomal orientations in the laboratory frame, $\hat{e}_{i}^{\prime}$, as well as $\left\langle C_{n}>\right.$ values calculated with cach new section platne. Plot shows the average error of labit plane recovery ats new section planes are added. Note how a decrease in $\left\langle C_{n}\right\rangle$ corresponds to a decrease in error due to increased randomness of sampling.

$\Delta g$ values and individual LMF and boundary-normal orientations. Since we do not simulate the detailed threedimensional microstructure, it is appropriate to substitute $l_{\Lambda}\left(\omega \mid \hat{e}_{3}^{s}, \Delta g\right)$ and $S_{v}\left(\hat{n}_{\Lambda} \mid \Delta g\right)$ with the number-fraction probability-distribution functions $n_{n}\left(\omega \mid \hat{e}_{3}^{\mathrm{s}}, \Delta g\right)$ and $N_{n}\left(\hat{n}_{A} \mid \Delta g\right)$. The trace normal distribution, $n_{n}\left(\omega \mid \hat{e}_{3}^{s}, \Delta g\right)$, is normalized by the total number of traces observed, and $N_{n}\left(\hat{n}_{A} \mid \Delta g\right)$ gives the number fraction of grain boundaries with the orientation $\hat{n}_{A}$. All other details of the stereological recovery are the same, with the exception that the factor of $\pi / 4$ is removed from the fundamental equation.

The first step in performing each simulation was to establish $N_{n}\left(\hat{n}_{A} \mid \Delta g\right)$ for a simulated microstructure. For most simulations, we assumed that $\Delta g$ was a general misorientation such that the domain of $N_{n}\left(\hat{n}_{A} \mid \Delta g\right)$ wals the hemisphere. All simulated $N_{n}\left(\hat{n}_{A} \mid \Delta g\right)$ functions consisted of random distributions plus two to eight "habit" planes of equal weight. The habit planes for each microstructure were chosen using 
pseudorandom numbers. After establishing $N_{n}\left(\hat{n}_{A} \mid \Delta g\right)$, it was necessary to specify the orientations of the LMFs associated with each bicrystal. For nontextured microstructures, LMF orientations with respect to the laboratory frame $\left(\hat{c}_{i}^{L}\right)$ were chosen randomly. For textured microstructures, LMF orientations were chosen by first randomly choosing a single LMF orientation as the "central" orientation. All LMF orientations were chosen randomly, but were required to fall within 15 deg of this central orientation. After specifying the LMFs we selected a grain-boundary plane for each bicrystal based on the established $N_{n}\left(\hat{n}_{A} \mid \Delta g\right)$ function, with no correlation between boundary type and LMF orientation. These steps completely constrained the microstructure.

After constraining the microstructure it was necessary to select the orientations of the section plane(s) with respect to the laboratory frame. In the case of random LMF distributions, a single section plane was used, but in the case of nonuniform LMF distributions, the seven section planes shown in Table II were used.

Having determined the microstructure and the sectionplane orientations, the next step was to calculate the "observed" traces, expressed in the GMF. Trace vectors were given by the intersections of all the grain-boundary planes and the section plane(s). In most simulations, the effect of trace-measurement error was simulated by rotating the observed trace in the plane of the section plane by an amount chosen randomly from an even distribution in the range of \pm 5 deg. In nontextured materials, a trace from each of the bicrystals was considered observed. In textured microstructures, we expect the sample texture to give rise to sampling bias for boundaries associated with the same section plane. This bias results from the fact that boundaries that are nearly parallel to the section plane are less likely to be observed than boundaries that are perpendicular to the section plane. The probability that a boundary will be cut by a section plane is proportional to $\sin (\alpha)$, where $\alpha$ is the angle between the grain-boundary plane and the section plane. For each section plane, a fraction of observed boundaries was chosen from a pool of "candidate" grain boundaries according to the probability $\sin (\alpha)$. Although the candidate grain boundaries were representative of $N_{n}\left(\hat{n}_{A} \mid \Delta g\right)$, the observed boundaries were not.

Once all traces were collected and expressed in the GMF according to these rules, $N_{n}\left(\hat{n}_{A} \mid \Delta g\right)$ was estimated using the new method. The parameter $\varepsilon$ was assigned to be 3 deg in all simulations. We chose to use $500 \mathrm{~S}$ frames, so that the average spacing between $\hat{e}_{3}^{S}$ directions was about $6 \mathrm{deg}$. The order $R$ of series truncation (Fig. 6) was usually taken to be 34 , but, in the cases of trace-measurement crror, better recoveries were obtained with $R=24$. The angular resolution was found to be about $1.5 \pi / R$.

For each recovered $N_{n}\left(\hat{n}_{A} \mid \Delta g\right)$ value, numerical integrations were performed in the regions surrounding the ten largest peaks in order to estimate the number fraction of boundaries at each peak. Known habit planes were then matched with recovered peaks, and the percent error in number fraction was calculated for cach peak. False peaks occurred when a recovered peak associated with no habit plane had a higher number fraction than a recovered peak that was associated with a habit plane. The data presented in Tables I and II demonstrate that the method may be used to estimate $S_{V}\left(\hat{n}_{A} \mid \Delta g\right)$ in a statistically reliable fashion. Moreover, simula- tions demonstrate that $S_{V}\left(\hat{n}_{A} \mid \Delta g\right)$ may be characterized on highly textured materials if several section planes are used. The simulations also demonstrate the usefulness of $\left\langle C_{n}\right\rangle$ in quantifying the randomness of available data.

\section{EXPERIMEN'TAL}

The proposed method was used to recover the function $S_{s}\left(\hat{n}_{\Lambda} \mid \Sigma 3\right)$ in 304 stainless steel. The sample was first treated in a vacuum furnace at $1010^{\circ} \mathrm{C}$ for 23.5 hours and oven cooled. A subsequent heat treatment at $600{ }^{\circ} \mathrm{C}$ for 4 hours "fine tuned" the boundaries to lower-energy configurations. ${ }^{\mid 15]}$

The sample was polished, and electron backscatter patterns taken from a FEI/PHILIPS* XL 30 scanning field-emission

*PHIL IPS is a trademark of Philips Electronics Corp., Mahwah, NJ.

gun were processed using Orientation Imaging Microscopy (OIM*) software. The step size of all scans was $2 \mu \mathrm{m}$. The

*OIM is a trademark of TSL, Inc., Draper, UT

average grain diameter was approximately $150 \mu \mathrm{m}$.

A grain-boundary reconstruction algorithm was used to find all boundary lengths, trace angles, and adjacent grain orientations. ${ }^{[16]}$ We estimated that in order to keep tracemeasurement error below $5 \mathrm{deg}$, only boundaries greater than 15 times the step size of the scans could be used in the analysis. However, boundaries of this length or greater accounted for only 59 pct of the total $\Sigma 3$ boundary trace lengths. For this reason, we collected the $S_{s}\left(\hat{n}_{A} \mid \Sigma 3\right)$ function with respect to all boundaries of length greater than $30 \mu \mathrm{m}$.

Figure 4 shows the function $S_{s}\left(\hat{n}_{A} \mid 23\right)$ taken from 717 bicrystals observed on four scans. For these bicrystals, $\left\langle C_{n}\right\rangle$ was found to be 0.0003 ; hence, we assumed that a single section plane would provide a reasonable estimate of $S_{S}\left(\hat{n}_{A}\right)$ 23). The largest peak is the coherent $\{111\} /\{111\}$ peak. An integration out to $9 \mathrm{deg}$ away from this peak returned $S_{s}$ $(\{111\} /\{111\} \mid \Sigma 3)=0.78$.

It is interesting to note similarities between Figures 4 and 5. Figure 5 is a plot of low-energy tilt boundaries on the $\langle 011\rangle$ zone that were observed in nickel by Randle. ${ }^{|14|}$ All boundary types observed by Randle were given equal weight in the plot of Figure 5. The plot is made to the 34 th

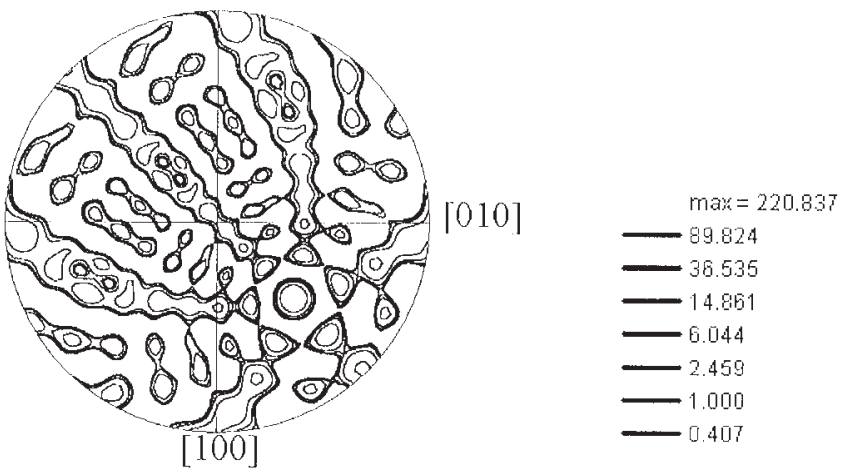

Fig. 4 Recovered $S_{3}\left(\Sigma 3, \hat{h}_{A}\right)$ for 717 boundaries of length greater than $30 \mu \mathrm{m}$. 


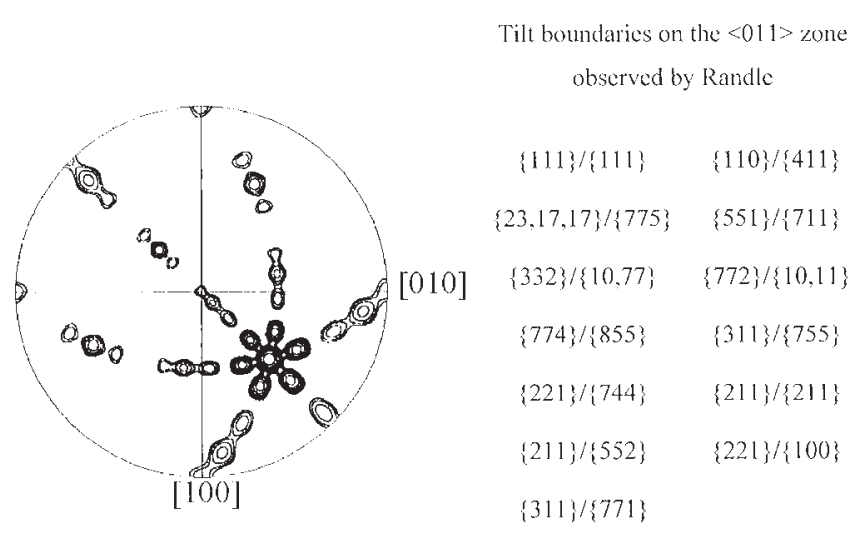

Fig. 5-Low-cnergy tilt boundarics on the $<011\rangle$ zone observed by Randle ef $a l^{\mid 151}$

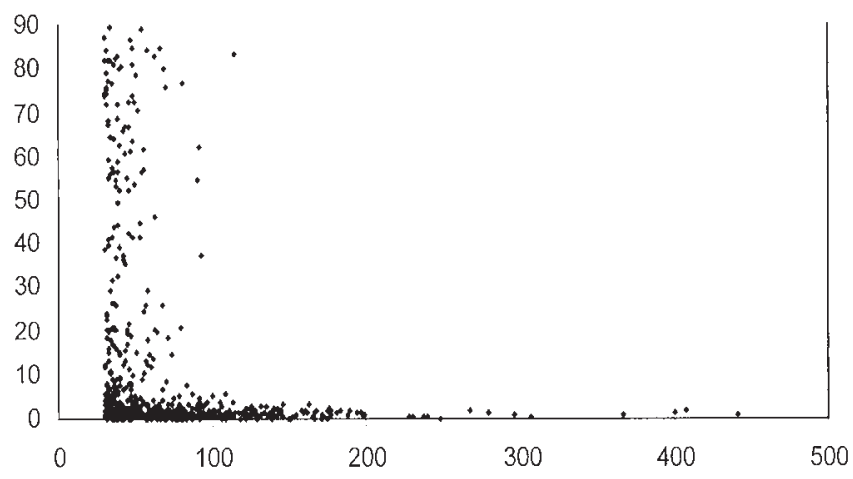

Fig. 6-Coherent trace deviation angle (in deg) vs trace length for 717 boundaries of length $>30$.

order. From a comparison of Figures 4 and 5 , it would seem that $\Sigma 3$ grain boundaries tend to align on the $\langle 011\rangle$ zone.

Figure 6 shows a plot of coherent trace-deviation angles of the same 717 boundaries as a function of their lengths (in microns). The coherent trace-deviation angle is the angle between the measured trace and the trace of the $\{111\} /\{111\}$ plane. ${ }^{|16,17|}$ It is likely that most grain boundaries with coherent trace-deviation angles near 0 deg are associated with coherent boundaries. Traces with coherent trace-deviation angles much greater than 0 deg cannot be coherent boundaries. The striking feature of Figure 6 is that the boundaries that are known to be noncoherent mostly occur at lengths smaller than about $60 \mu \mathrm{m}$. This result raises many fundamental questions about the grain-boundary planes of the small $\Sigma 3$ boundaries that, by number and area fraction, are a major $\Sigma 3$ boundary type.

In order to better understand what boundary types the noncoherent $\Sigma 3$ types were associated with, we decided to perform a stereological recovery only on the 146 boundaries with coherent trace-deviation angles greater than $10 \mathrm{deg}$. The result is shown in Figure 7 . By far, the three largest peaks from this recovery were $S_{s}(\{221\} /\{744\} \mid \Sigma 3)=0.39$, $S_{s}(\{111\} /\{511\} \mid \Sigma 3)=0.23$, and $S_{s}(\{211\} /\{211\} \mid \Sigma 3)=0.17$. It is interesting to note from Figure 7 the preponderance of grain-boundary planes near the $\{111\} /\{111\}$ position. Boundaries near $\{111\} /\{111\}$ are known to have lower energy than those removed from $\{111\} /\{111\}$.11 $^{11}$

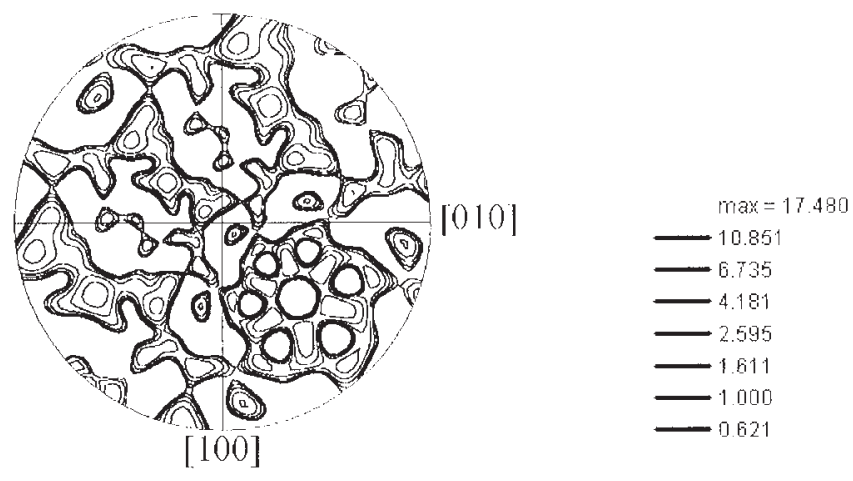

Fig. 7 -Recovered $S_{5}\left(\hat{n}_{4} \mid \Sigma 3\right)$ for 146 boundaries of length greater than 30 $\mu \mathrm{m}$ and coherent trace deviation angle greater than 10 deg.

\section{CONCLUSIONS}

We have derived the fundamental equation of a new stereology and have shown with both simulations and actual data that it may be used to recover grain-boundary plane distributions in the GMF. In the derivation of the new stereology, it was assumed that all boundary types are sampled in correct proportions and that all boundaries are sampled in a random manner. Even in the case of textured materials, it was shown that the method is reliable if multiple section planes are used. We have introduced the parameter $\left\langle C_{n}\right\rangle$ as an estimate of the randomness of the data and have shown it to be a reliable indicator of the accuracy of the recovery.

Trace measurements made from 304 stainless steel provided evidence that there is a tendency for 23 boundaries to be tilt boundaries on the $\langle 011\rangle$ yone. It was shown that a significant proportion of $\sum 3$ grain-boundary planes are of the smaller type, where coherent boundaries are less common. Measuring these boundary traces with enough precision to determine their trace angles accurately while still collecting many boundaries from a large area poses a significant experimental challenge. Nonetheless, the results obtained show the usefulness of quantifying the grain-boundary distribution through stereological methods.

\section{ACKNOWLEDGMENTS}

One of the authors (RJL) is grateful to the Office of Naval Research for fellowship support. BLA acknowledges the support of the NSF through the Materials Research Science and Engineering Center, Carnegie Mellon University (Grant No. DMR-0079996).

\section{APPENDIX}

\section{Derivation of $P(v)$}

Consider a grain-boundary plane whose normal lies $y$ deg from the $e_{3}^{\prime}$ direction. As stated previously, $P(v)$ is the probability that a randomly chosen trace angle from that boundary plane will lie within $\varepsilon$ deg of perpendicularity to the $\hat{e}_{3}^{S}$ direction. To obtain $P(v)$, it is assumed that all grain-boundary trace angles have the same probability of being chosen. 
From this assumption, it is clear that

$$
P(v)=\left(\frac{2 \gamma^{\prime}}{\pi}\right)
$$

where $2 \gamma^{\prime}$ is the range of trace angles on the boundary that have angulat deviations less than $\varepsilon$ from perpendicularity to the $\hat{s}$ direction, as shown in Figure Al.

From the clefinition of $P(v)$, it is obvious that $P(v)$ is completely independent of one's placement of $\hat{e}_{1}^{S}$ and $\hat{e}_{2}^{S}$. Without loss of generality, we are free to place $\hat{e}_{j}^{S}$ and $\hat{e}_{2}^{S}$ in a right-handed sense such that $\hat{e}_{j}^{S}$ is perpendicular to both the boundary normal, $\mathbf{n}_{(i, i}$, and the $\hat{e}_{3}^{S}$ direction, as shown in Figure Al.

In order to label all possible traces of the boundary, we begin by placing the grain-boundary normal parallel to the $\hat{e}$. direction. With the boundary normal in this posilion, we are free to place $\hat{e}_{i}^{S}$ anywhere within the boundary plane, since any choice of $\hat{e}_{i}^{Y}$ will be perpendicular to both the boundary normal and the $\hat{e}_{3}^{s}$ direction. The angle between a trace of the grain boundary and the $\hat{e}^{S}$ direction is given by $\gamma$. Now, we incline the boundary normal 10) $v$ degrees from the $\hat{e}_{3}^{S}$ axis by a rotation $R(v)$ of $v$ deg about the $-\hat{e}_{i}^{S}$ direction (where $0 \leq v \leq \pi / 2$ ). $R(v)$ is given by

$$
R(v)=\left[\begin{array}{ccc}
1 & 0 & 0 \\
0 & \cos v & \sin v \\
0 & -\sin v & \cos v
\end{array}\right]
$$

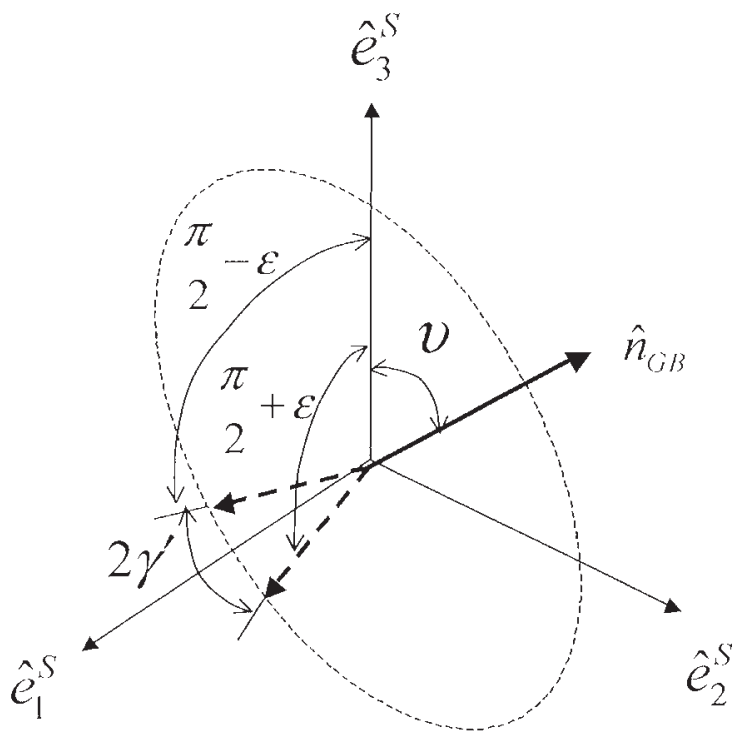

lijg. Al-2 $\gamma^{\prime}$ gives the range of grain-boundary traces that are within $\varepsilon$ deg of perpendicularily of the $\hat{r}_{3}$ direction.
We wish to find the two traces (with trace angles $\pm \gamma^{\prime}$ ) in the range $-\pi / 2<\gamma \leq \pi / 2$, for which the vectors $R(v)\left(\begin{array}{c}\cos \left(\gamma^{\prime}\right) \\ \sin \left( \pm \gamma^{\prime}\right) \\ 0\end{array}\right)$ from angles of $\pi / 2 \mu \pm \varepsilon$ with the $\hat{e}_{3}^{s}$ axis. These traces correspond to the dashed vectors shown in Figure Al. Any trace such that $|\gamma| \leq \gamma^{\prime}$ will have an angular deviation less than $\varepsilon$ from perpendicularity to the $\hat{e}_{3}^{S}$ direction. Using the dot product, we write

$$
\cos \left(\frac{\pi}{2} \mu \pm \varepsilon\right)=(001) \cdot R(v)\left(\begin{array}{c}
\cos \left(\gamma^{\prime}\right) \\
\sin \left( \pm \gamma^{\prime}\right) \\
0
\end{array}\right)
$$

from which we obtain

$$
\sin \varepsilon=\sin v \sin \gamma^{\prime}
$$

Combining Eqs. [Al] and [A4], we have

$$
P(v)=\frac{2}{\pi} \sin ^{-1}\left(\frac{\sin (\varepsilon)}{\sin (v)}\right) \quad \text { when } v \geq \varepsilon
$$

When $v<\varepsilon, P(v)$ is undefined in the preceding expression. For this case, it is clear that

$$
P(v)=1 \quad \text { when } v<\varepsilon
$$

\section{REFERENCES}

1. D. Wolf and K.L. Merkle: in Materials Interfaces: Atomic-Level Structure and Properties, D. Wolf and S. Yip, eds. Chapman \& Hall, I.ondon, 1992, pp. 87-150.

2. F.A. Trillo and L.E. Murr: Acta Mater, 1999, vol. 47, p. 235.

3. J.E. Hilliard: Trans. AlME, 1962, vol. 224, p. 1201.

4. B.L. Adams: Metall. Trans. A, 1986, vol. 17A, pp. 2199-2207.

5. A. Morawice: in Grain Growth in Polycrystalline Materials III, H. Weiland, B.L. Adams, and A.D. Rollett, eds., TMS, Warrendale, PA, 1998, pp. 509-14.

6. R.J. Larsen and B.L. Adams: Plasticity, Damage and Fracture at Macro, Micos and Nano Scales: Pioc. Plasticity '02: 9h lnt. Symp. on Plasticity and th Current Applications, A.S. Kahn and O. Lopez-Pamics, cds., Ncat Press, Fulton, MD, 2002, pp. 27-29.

7. R.J. Iarsen: Master's 'Thesis, Brigham Young University, Provo, UT, 2002 .

8. D.M. Saylor and G.S. Rohrer: J. Am. Ceram. Soc., 2002, vol. 85, pp. 2799-2804

9. D.M. Saylor, B.S. El-Dasher, B.L. Adams, and G.S. Rohrer: Metall. Mater. Trans. A, 2004, vol. 35A, pp. $1981-89$.

I0. H.J. Bunge: Texture Analysis in Materials Science, Butterworth and Co., London, 1982.

11. A. Morawice and D.P. Field: Phil. Mag., 1996, vol. A73 (4), p. 1113.

12. D.M. Saylor: Ph.D. Thesis, Carnegie Mellon University, Pittsburgh, PA, 2001.

13. E.R. Wiebel: Stereological Methods Vol. 2: Theoretical Foundations, Academic Press, London, 1980.

14. D.P. Field and B.I. Adams: Metall, Trans. A, 1992, vol. 23A, pp. $251.5-26$.

15. V. Randle, P. Davies, and B. Hulm: Phil. Mag., 1999, vol. A79, p. 305 .

16. S.I. Wright and R.J. Iarsen: J. Microsc, 2002, vol. 205, p. 245.

17. V. Randle: Scripta Mater, 2001, vol, 44, p. 2789 